B. L. Rozovskii and A. Shimizu Nagoya Math. J.

Vol. 84 (1981), 195-208

\title{
SMOOTHNESS OF SOLUTIONS OF STOCHASTIC EVOLUTION EQUATIONS AND THE EXISTENCE OF A FILTERING TRANSITION DENSITY
}

\section{B. L. ROZOVSKII AND A. SHIMIZU}

In this paper, we shall discuss the smoothness of solutions of stochastic evolution equations, which has been investigated in N. V. Krylov and B. L. Rozovskii [2] [3], to establish the existence of a filtering transition density.

First, we introduce the filtering equation, which has been discussed in [1] [3] [6] and [9]. Let us consider the system $\left(x_{t}, y_{t}\right)$ given by the stochastic differential equation

$$
\begin{aligned}
& d x_{t}=a\left(x_{t}, y_{t}, t\right) d t+b\left(x_{t}, y_{t}, t\right) d \nu_{t} \\
& d y_{t}=A\left(x_{t}, y_{t}, t\right) d t+B\left(y_{t}, t\right) d \nu_{t} \\
& x_{0}=\theta, y_{0}=\xi, t \in[0, T], T<+\infty,
\end{aligned}
$$

where $\nu=\left\{\nu_{t}\right\}_{t \in[0, T]}$ is a $\left(d+d_{1}\right)$-dimensional Brownian motion defined on a complete probability space, and $a, A, b$ and $B$ are matrices of type $d \times 1, d_{1} \times 1, d \times\left(d+d_{1}\right)$ and $d_{1} \times\left(d+d_{1}\right)$ respectively. We denote by $F_{t}^{Y}$ the complete $\sigma$-algebra $\sigma\left\{y_{t}, 0 \leqq \tau \leqq t\right\}$. Let us denote by $P_{t}[f]$ a measurable modification of the conditional expectation $E\left[f\left(x_{t}, y_{t}, t\right) \mid F_{t}^{\mathrm{Y}}\right]$. We put

$$
\begin{aligned}
& C=\left(B B^{*}\right)^{-1 / 2}, \quad \beta(x, y, t)=C A, \\
& \bar{w}_{t}=\int_{0}^{t} C\left(y_{\tau}, \tau\right) d y_{\tau}-\int_{0}^{t} P_{\tau}[\beta] d \tau, \\
& y_{t}^{\prime}=\bar{w}_{t}+\int_{0}^{t} P_{\tau}[\beta] d \tau
\end{aligned}
$$

and

$$
\rho_{t}=\exp \left\{-\int_{0}^{t} P_{\tau}[\beta] d \bar{w}_{\tau}-\frac{1}{2} \int_{0}^{t}\left|P_{\tau}[\beta]\right|^{2} d \tau\right\}
$$

Received March 11, 1980. 
Here, $|\cdot|$ denotes the norm in $R^{d_{1}}$ and $\widetilde{w}_{t}$ is a $d_{1}$-dimensional $F_{t}^{Y}$-Brownian motion. Under the assumptions $\left.A_{1}\right)-A_{6}$ ) in $\S 1$ in [3], it is known that $\Phi_{t}[\eta]=P_{t}[\eta] \rho_{t}^{-1}, \eta \in C_{0}^{\infty}\left(R^{d}\right)$, satisfies the next equation

$$
d \Phi_{t}[\eta]=\Phi_{t}[L \eta] d t+\Phi_{t}[M \eta] d y_{t}^{\prime},
$$

where $C_{0}^{\infty}\left(R^{d}\right)$ denotes the space of $C^{\infty}$-functions with compact support, $L$ is a differential operator of second order, $M$ is a differential operator of first order, and the coefficients of the both operators $L$ and $M$ depend on $t, x$ and $\omega$. To state Equation (0.1) precisely, we need some preliminaries. We denote by $a_{i j}(x, y, t)$ the $(i, j)$-component of the matrix $\frac{1}{2} b(x, y, t) b *(x, y, t)$, $a_{i}(x, y, t)$ denotes the $i$-th component of the vector $a$ and $\beta_{k}(x, y, t)$ means the $k$-th component of the vector $\beta(x, y, t)$. Put

$$
\alpha(x, y, t)=C(y, t) B(y, t) b^{*}(x, y, t),
$$

and let $\alpha_{i j}(x, y, t)$ be the $(i, j)$-component of the matrix $\alpha(x, y, t)$. Then, the operators $L$ and $M$ can be written as follows:

$$
\begin{aligned}
L \eta & =\sum_{i=1}^{d} a_{i}\left(x, y_{t}, t\right) \frac{\partial}{\partial x_{i}} \eta+\sum_{i, j=1}^{d} a_{i j}\left(x, y_{t}, t\right) \frac{\partial^{2}}{\partial x_{i} \partial x_{j}} \eta, \\
M \eta & =\left(M_{1} \eta, M_{2} \eta, \cdots, M_{d_{1}} \eta\right)
\end{aligned}
$$

and

$$
M_{k} \eta=\beta_{k}\left(x, y_{t}, t\right) \eta+\sum_{i=1}^{d} \alpha_{k i}\left(x, y_{t}, t\right) \frac{\partial}{\partial x_{i}} \eta, \quad\left(k=1,2, \cdots, d_{1}\right) .
$$

In this paper, we shall study the Cauchy problem of Equation (0.1) with the initial condition

$$
\Phi_{s}[\eta]=\eta(z) \quad \text { for all } \eta \in C_{0}^{\infty}\left(R^{d}\right),
$$

where $z$ is an arbitrarily fixed point in $R^{d}$. Making use of Sobolev's lemma (Theorem 2.2 in [3]), we can see that there exists a process $\psi$. in a space $\hat{H}_{2}^{] d / 2[}(s, T)$, which will be defined in $\S 1$, such that

$$
\Phi_{t}[\eta]=\left(\psi_{t}, \eta\right)_{] d / 2[},
$$

where ] $d / 2\left[\right.$ is the smallest natural number bigger than $d / 2$, and $(\cdot, \cdot)_{] d / 2[}$ is the inner product of the Sobolev space $W_{2}^{] \alpha / 2[}\left(R^{d}\right)$. Hence, Equation (0.1) with initial condition (0.2) can be written in the form

$$
\left(\psi_{t}, \eta\right)_{l}=(\gamma, \eta)_{l}+\int_{s}^{t}\left(\psi_{\tau}, L \eta\right)_{l} d \tau+\int_{s}^{t}\left(\psi_{\tau}, M \eta\right)_{t} d y_{\tau}^{\prime},
$$


where $l=] d / 2\left[{ }^{*}\right) \quad$ By the Girsanov's theorem we see that Equation (0.3) can be regarded as follows:

$$
\left(\psi_{t}, \eta\right)_{l}=(\gamma, \eta)_{l}+\int_{s}^{t}\left(\psi_{\tau}, L \eta\right)_{l} d \tau+\int_{s}^{t}\left(\psi_{\tau}, M \eta\right)_{l} d w_{\tau}
$$

Here,

$$
\begin{aligned}
\int_{s}^{t}\left(\psi_{\tau}, M \eta\right)_{l} d w_{\tau} & =\sum_{k=1}^{d_{1}} \int_{s}^{t}\left(\psi_{\tau}, M_{k} \eta\right)_{l} d \omega_{\tau}^{k}, \\
& \text { where } w_{t}=\left(w_{t}^{k}\right)_{k=1,2, \ldots, d_{1}} \text { is a } F_{t}^{y} \text {-Brownian motion } .
\end{aligned}
$$

From $\S 1$, the $\sigma$-algebra $F_{t}^{Y}$ will be denoted by $F_{t}$ for simplicity, and $w_{t}$ will be replaced by the notation $B_{t}$.

We shall make the same assumptions on the coefficients of the operators $L$ and $M$ as in [2], which will be stated in $\S 1$. By a solution of Equation (0.4), we mean a function $\psi_{0} \in \hat{H}_{2}^{l}(s, T)$ with values in $W_{2}^{l}\left(R^{d}\right)$ for each $(t, \omega)$ and finite $E\left[\left\|\psi_{t}\right\|_{l}^{2}\right]^{* *)}$ for all $t$, which satisfies Equation (0.4) for any $\eta \in C_{0}^{\infty}\left(R^{d}\right)$ and $t \in[s, T]$ with probability 1 . N. V. Krylov and B. L. Rozovskii [2] investigated the existence, the uniqueness and the smoothness of solutions of Equation (0.4). In the introduction, we will state our results for simplicity only in the case that the coefficients of $L$ and $M$ are sufficiently smooth. That is, in addition to the assumptions in [2], we assume here in the introduction that the coefficients of $L$ and $M$ are $C^{\infty}$-functions in $x$ for each $(t, \omega)$, and that their derivatives do not exceed a constant $K$ in absolute value for any $(t, x, \omega)$ and $\alpha$. In this case, the Krylov-Rozovskii's result on the smoothness of solutions (Theorem 2.2 in [2]) can be stated as follows: Let $m \geqq 0$. If $E\left[\|\gamma\|_{2 l+m}^{2}\right]$ is finite, then the solution $\psi$. of Equation (0.4) belongs to the space $\hat{H}_{2}^{2 l+m+1}(s, T)$. In this statement, it should be noticed that the smoothness of the solution depends on the smoothness of the initial data $\gamma$. Under the initial condition (0.2), we can assume $\gamma \in W_{2}^{l}\left(R^{d}\right)$, but we can not suppose further smoothness of $\gamma$. Therefore, we should remove the condition on the smoothness of $\gamma$ to discuss the smoothness of the solution $\psi_{t}$. Our result on the smoothness of the solution of Equation (0.4) can be formulated as follows: The solution $\psi$. of Equation (0.4) satisfies $\psi_{t}(x, \omega) \in \bigcap_{m} W_{2}^{m}\left(R^{d}\right)$ for each $(t, \omega) \in(s, T] \times \Omega$. Since we obtain this result without the smoothness of $\gamma$, we shall obtain the following expression for the solution $\Phi^{z}$. of Equation (0.1) with initial condition (0.2): $\Phi^{z}$ can be expressed in the form

*) $\gamma$ is a function in $W_{2}^{l}\left(R^{d}\right)$ such that $\eta(z)=(\gamma, \eta)_{l}$.

**) $\|\cdot\|_{\imath}$ denotes the norm of the Sobolev space $W_{2}^{l}\left(R^{d}\right)$. 


$$
\Phi_{t}^{z}[\eta]=\int_{R^{d}} \phi(s, z, t, x ; \omega) \eta(x) d x, \quad(t, z, x) \in(s, T] \times R^{d} \times R^{d},
$$

for any $\eta \in C_{0}^{\infty}\left(R^{d}\right)$, where $\phi(s, z, t, x ; \omega)=(I-\Delta)^{l} \psi_{t}, I$ is the identity operator and $\Delta$ is the Laplace operator.

\section{§1. Notation and results}

Let $R^{d}$ be the $d$-dimensional Euclidean space, $T$ a fixed positive number, $(\Omega, F, P)$ a complete probability space $\left\{F_{t}\right\}_{t \in[0, T]}$ an increasing family of complete $\sigma$-algebras contained in $F, d_{1}$ a positive integer, and $\left\{B_{t}\right\}_{t \in[0, T]}$ a $d_{1}$-dimensional $F_{t}$-Brownian motion. We shall fix a basis in $R^{a}$ and denote by $\alpha, \alpha_{i}, \beta, \gamma \cdots$ arbitrary unit coordinate vectors as well as the zero vector. If $\alpha=0$, then $D^{\alpha}$ denotes the identity operator, while if $\alpha$ is the $i$-th basis vector, then $D^{\alpha}=\partial / \partial x^{i}$.

We suppose that the coefficients $\mathrm{a}_{i j}\left(x, y_{t}, t\right), \alpha_{k j}\left(x, y_{t}, t\right)$ of the operators $L$ and $M$ are differentiable in $x$, then it is obvious that the operators $L$ and $M$ can be written as follows.

$$
L \eta=(-1)^{|\alpha|+|\beta|} D^{\beta}\left(a_{t}^{\alpha \beta}(x) D^{\alpha} \eta\right)
$$

and

$$
M \eta=(-1)^{|\alpha|} D^{\alpha} a_{t}^{\alpha}(x),
$$

where $a_{t}^{\alpha \beta}(x)$ are real functions defined on $[0, T] \times R^{d} \times \Omega, a_{t}^{\alpha}(x)$ are functions with values in $R^{d}$ defined on the same space, $|\cdot|$ is the norm of the $d$-dimensional Euclidean space, and the argument $\omega$ is omitted as a rule. Here and throughout the paper, the summation convention is in force for repeated indices. $W_{2}^{k}\left(R^{d}\right)$ is the Sobolev space of all real functions $\psi$ defined on $R^{d}$ with finite norm

$$
\|\psi\|_{k}=\left(\sum_{\alpha_{1}, \cdots, \alpha_{k}}\left\|D^{\alpha_{1}} \cdots D^{\alpha_{k}} \psi\right\|^{2}\right)^{1 / 2}
$$

where $D^{\alpha_{1}} \cdots D^{\alpha_{k}}$ are generalized derivatives of $\psi$, and $\|\cdot\|$ denotes the norm of the space $L^{2}\left(R^{d}\right)$.

We will mention the assumptions on the coefficients $a_{t}^{\alpha \beta}(x), a_{t}^{\alpha}(x)$ of the operators $L$ and $M$. We denote by $B([0, T])$ the Borel sets in $[0, T]$, and by $B\left(R^{d}\right)$ the Borel sets in $R^{d}$. For the rest of this paper, we shall make the following assumptions:

Let us fix positive constants $K, \delta$ and an integer $m \geqq 0$. 
A) The functions $a_{t}^{\alpha \beta}(x)$ and $a_{t}^{\alpha}(x)$ are $B([0, T]) \times B\left(R^{d}\right) \times F$-measurable, and they are $F_{t}$-measurable for each $(t, x)$.

B) For all $x, \xi \in R^{d}, t \in[0, T]$ and $\omega \in \Omega$, the inequality

$$
2 \sum_{|\alpha|=|\beta|=1} a_{t}^{\alpha \beta}(x) \xi^{\alpha} \xi^{\beta}-\left|\sum_{|\alpha|=1} a_{t}^{\alpha}(x) \xi^{\alpha}\right|^{2} \geqq \delta|\xi|^{2}
$$

holds where $\xi^{\alpha}$ is the $i$-th coordinate of $\xi$ if $\alpha$ is the $i$-th coordinate vector.

C) The functions $a_{t}^{\alpha \beta}(x), a_{t}^{\alpha}(x)$ and their derivatives in $x$ up to $m$ inclusive are continuous in $x$ for each $(t, \omega)$; these functions and their derivatives do not exceed a constant $K$ in absolute value (in length, for the vectors $\left.a_{t}(x)\right)$ for any $(t, x, \omega)$ and $\alpha$.

Let $s$ be a real number such that $s \in[0, T]$, and we will introduce the spaces $H_{2}^{k}(s, T)$ and $\hat{H}_{2}^{k+1}(s, T)$. We denote by $H_{2}^{k}(s, T)$ the space of $L^{2}\left(R^{d}\right)$-valued functions $\psi=\psi_{t}(\omega)$ defined on $[s, T] \times \Omega$ such that

1) $\psi_{t}(\omega)$ is measurable in $(t, \omega)$, and for each $t$ it is $F_{t}$-measurable in $\omega$.

2) $\psi_{t}(\omega) \in W_{2}^{k}\left(R^{d}\right)$ for almost all $(t, \omega)$, and

$$
\left\|\psi_{.}\right\|_{k}=\left(E\left[\int_{s}^{T}\left\|\psi_{t}\right\|_{k}^{2} d t\right]\right)^{1 / 2}<+\infty .
$$

Here, measurability of $\psi=\psi_{t}(\omega)$ is understood in the sense of measurability of functions with values in a metric space $L^{2}\left(R^{d}\right)$. It is well-known that this measurability is equivalent both to strong and to weak measurability.

By $\hat{H}_{2}^{k+1}(s, T)$ we denote the subspace of $H_{2}^{k+1}(s, T)$ consisting of functions $\psi=\psi_{t}(\omega)$ with values in $W_{2}^{k}\left(R^{d}\right)$ for each $(t, \omega) \in[s, T] \times \Omega$, such that $E\left[\left\|\psi_{t}\right\|_{k}^{2}\right]<+\infty$ for all $t \in[s, T]$. Let us consider the next stochastic evolution equation

$$
\begin{aligned}
\left(\psi_{t}, \eta\right)_{n}= & (\gamma, \eta)_{n}+\int_{s}^{t}\left(D^{\beta} \psi_{\tau},(-1)^{|\alpha|} a_{\tau}^{\alpha \beta}(x) D^{\alpha} \eta\right)_{n} d \tau \\
& +\int_{s}^{t}\left(D^{\alpha} \psi_{\tau}, a_{\tau}^{\alpha}(x) \eta\right)_{n} d B_{\tau}+\int_{s}^{t}\left(f_{\tau}, \eta\right)_{n} d \tau,
\end{aligned}
$$

where scalar products are intended by the notation in the third term in the right hand side, and $n=l+1$.

From now on, we assume that the coefficients $a_{t}^{\alpha}(x)$ for $|\alpha|=1$ are $(n+1)$-times continuously differentiable in $x$ for each $(t, \omega)$, and that their derivatives in $x$ do not exceed a constant $K$ in length for any $(t, x, \omega)$ 
and $\alpha$. By a solution of Equation (1.1), we understand a function $\psi$. $\epsilon$ $\hat{H}_{2}^{n+1}(s, T)$ which satisfies Equation (1.1) for any $\eta \in C_{0}^{\infty}\left(R^{d}\right)$ and $t \in[s, T]$ with probability 1 .

We should give a notice that the equality

$$
\left(\Lambda^{-j} v, \eta\right)_{j+k}=(v, \eta)_{k}, \quad \Lambda=I-\Delta,
$$

holds for any $v \in W_{2}^{k}\left(R^{d}\right)$ and $\eta \in W_{2}^{j+k}\left(R^{d}\right)$ if $j$ is an integer and $k$ is a non-negative integer such that $j+k \geqq 0$. When $f=0$ in (1.1), we can see by (1.2) that a process $\psi$. with initial data $\gamma$ satisfies (0.4) if and only if $\Lambda^{-1} \psi$. with initial data $\Lambda^{-1} \gamma$ satisfies (1.1).

Then, we shall obtain

TheOREM 1. Let $n \leqq m$. Fix a non-negative integer $k$ such that $n+$ $k \leqq m$. Suppose that $a_{t}^{\alpha}(x)$ for $|\alpha|=1$ have derivatives of order $n+k+1$ with respect to $x$ which are continuous in $x$ for each $(t, \omega)$ and uniformly bounded in $\alpha, t, x$ and $\omega$ in the sense of length of d-dimensional vectors, and that $\gamma=\gamma(x, \omega)$ belongs to $W_{2}^{n+k}\left(R^{d}\right)$ for each $\omega$ and $E\left[\|\gamma\|_{n+k}^{2}\right]$ is finite. Besides, if $f . \in \hat{H}_{2}^{n+k}(s, T)$, then Equation (1.1) has a solution $\psi_{.} \in \hat{H}_{2}^{n+k+1}(s, T)$. Especially when $k=0$ and $f=0$, Equation (1.1) has a solution, and hence Equation (0.4) has a solution $\psi . \in \hat{H}_{2}^{n+1}(s, T)$.

Here, we will write the results on the uniqueness of solutions which have been obtained in [2].

Proposition 1 ([2]; Theorem 2.1). Let $n \leqq m$. We assume that $a_{t}^{\alpha}(x)$ for $|\alpha|=1$ have derivatives of order $n+1$ with respect to $x$ which are continuous in $x$ for each $(t, \omega)$ and uniformly bounded in $\alpha, t, x$ and $\omega$ in the sense of length of vectors. Let $\psi^{1}$. and $\psi^{2}$. be solutions of Equation (1.1). Then $E\left[\left\|\psi_{t}^{1}-\psi_{t}^{2}\right\|_{n}^{2}\right]=0$ holds for all $t \in[s, T]$.

Proposition 2 ([2]; Corollary 2.1). Let $l+1 \leqq m$. Suppose that $a_{t}^{\alpha}(x)$ for $|\alpha|=1$ have derivatives of order $l+2$ with respect to $x$ which are continuous in $x$ for each $(t, \omega)$ and uniformly bounded in $\alpha, t, x$ and $\omega$ in the sense of length of vectors. Let $\psi^{1}$. and $\psi^{2}$. be solutions of Equation (0.4). Then, $E\left[\left\|\psi_{t}^{1}-\psi_{t}^{2}\right\|_{l}^{2}\right]=0$ holds for all $t \in[s, T]$.

Making use of Theorem 1 and Proposition 1, we shall obtain

TheOREM 2. Let $n \leqq m$. Suppose that $a_{t}^{\alpha}(x)$ for $|\alpha|=1$ have derivatives of order $n+1$ with respect to $x$ which are continuous in $x$ for each $(t, \omega)$ and uniformly bounded in $\alpha, t, x$ and $\omega$ in the sense of length of vectors, 
and that $\gamma=\gamma(x, \omega)$ belongs to $W_{2}^{l}\left(R^{d}\right)$ for each $\omega$ and $E\left[\|\gamma\|_{l}^{2}\right]$ is finite. Then, Equation (0.4) has a solution $\psi$. such that $\psi_{t}(x, \omega) \in W_{2}^{m-2}\left(R^{d}\right)$ for each $(t, \omega) \in(s, T] \times \Omega$.

Theorem 2 and Proposition 2 imply

Corollary to Theorem 2. Let $2 n \leqq m$. Suppose that $a_{t}^{\alpha}(x)$ for $|\alpha|=$ 1 have derivatives of order $n+2$ with respect to $x$ which are continuous in $x$ for each $(t, \omega)$ and uniformly bounded in $\alpha, t, x$ and $\omega$ in the sense of length of vectors. Then, the Cauchy problem of Equation (0.1) with initial condition $(0.2)^{*)}$ has a unique solution $\Phi_{t}^{z}[\cdot]$, which can be expressed in the form

$$
\Phi_{t}^{z}[\eta]=\int_{s}^{t} \phi(s, z, t, x ; \omega) \eta(x) d x, \quad t \in(s, T],
$$

where $\phi(s, z, t, x ; \omega) \in W_{2}^{m-2 n}\left(R^{d}\right)$ for $(z, t, \omega) \in R^{d} \times(s, T] \times \Omega$ as a function of $x$.

Remark. Theorem 2 can be regarded as an extension of the existence and smoothness theorem of fundamental solutions of parabolic differential equations, whose proof has been given in [8].

\section{§2. Proof of Theorem 1}

First, we should give the preliminaries of the proof. We will give a quick review on the result obtained by N. V. Krylov and B. L. Rozovskii in [4] and [5]. Here, their result will be given in a simpler case than in [4] and [5].

Let $H$ be a real separable Hilbert space, and let $V$ be a reflexive real separable Banach space. $\left\{B_{t}\right\}_{t \in[0, T]}$ denotes a $F_{t}$-Brownian motion with values in the $d$-dimensional Euclidean space $R^{d}$ (In [4] and [5], the space $R^{d}$ was replaced by a real separable Hilbert space $E$.). Suppose that $V$ $\subset H=H^{*} \subset V^{*}$, that the imbedding mappings are dense and continuous, and that $\left\langle v^{*}, v\right\rangle=\left(v^{*}, v\right)^{* *)}$ if $v^{*} \in H$. Here, $V^{*}\left(H^{*}\right)$ denotes the space

*) By a solution of the Cauchy problem (0.1) with (0.2), we mean a real valued function $\Phi_{t}[\eta]$ defined on $[s, T] \times \Omega \times C\left(R^{d}\right)$ such that (i) for each $(t, \omega) \in[s, T] \times \Omega, \Phi_{t}[\eta]$ is a linear fnnctional on $C\left(R^{d}\right)$, the space of all bounded continuous functions on $R^{d}$, satisfying $\left|\Phi_{t}[\eta]\right| \leqq \tilde{\rho}_{t}^{-1} \sup _{x \in R^{d}}|\eta(x)|$ for $(t, x, \eta) \in[s, T] \times \Omega \times C\left(R^{d}\right)$, where $\tilde{\rho}_{t}$ is a version of $\rho_{t}$ continuous for all $\omega \in \Omega$, (ii) for each $\eta, \Phi_{t}[\eta]$ is $(t, \omega)$-measurable, (iii) for each $(t, \eta), \Phi_{t}[\eta]$ is $F_{t}$-measurable, (iv) for each $(\omega, \eta), \Phi_{t}[\eta]$ is $t$-continuous and (v) $\Phi_{t}[\eta]$ satisfies $(0.1)$ and (0.2).

**) $(\cdot, \cdot)$ denotes the inner product of the Hilbert space $H$. 
of linear functionals on $V$ (resp. $H$ ), and $\left\langle v^{*}, v\right\rangle$ means the value of $v^{*} \in V^{*}$ at $v \in V$.

Let $A(\cdot, t, \omega)$ and $\boldsymbol{B}(\cdot, t, \omega)=\left(\boldsymbol{B}^{i}(\cdot, t, \omega)\right)_{i=1, \ldots, d_{1}}$ be mappings defined on $V$ with values in $V^{*}$ and $H^{d_{1}}=H \times H \times \cdots \times H\left(d_{1}\right.$-fold) respectively for all $(t, \omega)$. For each $v \in V$, the functions $A(v, t, \omega)$ and $B(v, t, \omega)$ are measurable in $(t, \omega)$ (with respect to the $d t \times d P$-completed algebra), and $F_{t^{-}}$ measurable in $\omega$ for fixed $t \in[s, T]$.

Let us consider the following Itô's equation

$$
u_{t}=u_{s}+\int_{s}^{t} A\left(u_{\tau}, \tau, \omega\right) d \tau+\int_{s}^{t} \boldsymbol{B}\left(u_{\tau}, \tau, \omega\right) d B_{\tau}, \quad t \in[s, T],
$$

where $\boldsymbol{B}\left(u_{\tau}, \tau, \omega\right) d B_{r}$ in the right hand side in (2.1) means the inner product. A function $u_{t}(\omega)$ with values in $H$, defined on $[s, T] \times \Omega$ is called a solution of Equation (2.1) if it is measurable in $(t, \omega)$ and $F_{t}$-measurable for each $t \in[s, T]$, and if it belongs to $L^{2}([s, T] \times \Omega, V) \cap L^{2}(\Omega, C([s, T] ; H))$ and satisfies Equation (2.1) (as an equation in $V^{*}$ ) for all $t \in[s, T]$ with probability 1 .

We assume the following conditions (A.I)-(A.IV) on the functions $A(v, t, \omega)$ and $\boldsymbol{B}(v, t, \omega)$, which will be denoted by $\boldsymbol{A}(v)$ and $\boldsymbol{B}(v)$ respectively.

$$
\left\langle A\left(v^{1}+\lambda v^{2}\right), v\right\rangle \text { is continuous in } \lambda \in R^{1} .
$$

There exist positive constants $N, \varepsilon$ and a non-negative function $f(t, \omega)$ defined on $[s, T] \times \Omega, F_{t}$-measurable for each fixed $t \in[s, T]$, belonging to $L^{1}([s, T] \times \Omega)$ such that for all $v, v^{1}$ and $v^{2} \in V,(t, \omega) \in[s, T] \times \Omega$ are satisfied the next statements (A.II)-(A.IV):

$$
\begin{array}{ll}
\text { (A.II) } & 2\left\langle A\left(v^{1}\right)-A\left(v^{2}\right), v^{1}-v^{2}\right\rangle+\sum_{i=1}^{d_{1}}\left\|\boldsymbol{B}^{i}\left(v^{1}\right)-\boldsymbol{B}^{i}\left(v^{2}\right)\right\|_{H}^{2} \leqq N\left\|v^{1}-v^{2}\right\|_{H}^{2}, \\
\text { (A.III) } & 2\langle A(v), v\rangle+\sum_{i=1}^{d_{1}}\left\|\boldsymbol{B}^{i}(v)\right\|_{H}^{2} \leqq-\varepsilon\|v\|_{V}^{2}+f(t, \omega)+N\|v\|_{H}^{2}, \\
\text { (A.IV) } & \|\boldsymbol{A}(v)\|^{*} \leqq f(t, \omega)^{1 / 2}+N\|v\|_{V} .
\end{array}
$$

Then, the next proposition has been established in [4] and [5] (Theorem 1 in [4], Corollary II.2.1 and Theorem II.2.2 in [5]).

Proposition. Under the assumptions (A.I)-(A.IV) and given initial data $u_{s} \in H$, there exists a solution $u_{t}$ of Equation (2.1). Let $u_{t}^{1}$ and $u_{t}^{2}$ be solutions of Equation (2.1), then $E\left[\left\|u_{t}^{1}-u_{t}^{2}\right\|_{H}^{2}\right]=0$ for each $t \in[s, T]$.

The next lemma will be used to introduce operators $A_{t}$ and $B_{t}$ in the proof of Theorem 1. Since the proof of Lemma is elementary, it will be omitted. 
LEMma. Under the assumptions of Theorem 1, the following equalities hold:

$$
\begin{aligned}
& \left(D^{\beta} \psi,(-1)^{|\alpha|} a_{t}^{\alpha \beta} D^{\alpha} \eta\right)_{n}=\left(a_{t}^{\alpha \beta} D^{\beta} D^{\alpha_{1}} D^{\alpha_{2}} \cdots D^{\alpha_{n}} \psi,(-1)^{|\alpha|} D^{\alpha} D^{\alpha_{1}} \cdots D^{\alpha_{n}} \eta\right)_{0} \\
& +\sum_{j=1}^{n} \sum_{\substack{\alpha_{1} \\
\left|\alpha_{i_{1}}\right| \cdots \cdots, \ldots=\left|\alpha_{n}\right|=1}}\left(b^{\alpha_{i_{1}} \alpha_{i_{2}} \cdots \alpha_{i_{j}} \alpha_{\beta}} D^{\beta} D^{\alpha_{1}} \cdots D^{\alpha_{n}} \psi,(-1)^{|\alpha|}\right. \\
& \left.\times D^{\alpha} D^{\alpha_{1}} \cdots \hat{D}^{\alpha_{i_{1}}} \cdots \hat{D}^{\alpha_{i_{j}}} \cdots D^{\alpha_{n} \eta}\right)_{0}^{*}
\end{aligned}
$$

for any $\psi$ and $\eta \in W_{2}^{n+1}\left(R^{d}\right)$, and

$$
\begin{aligned}
& \left(D^{\alpha} \psi, a_{t}^{i \alpha} \eta\right)_{n}=\left(a_{t}^{i \alpha} D^{\alpha} D^{\alpha_{1}} \cdots D^{\alpha_{n}} \psi, D^{\alpha_{1}} \cdots D^{\alpha_{n}} \eta\right)_{0} \\
& +\sum_{j=1}^{n} \sum_{\substack{\alpha \alpha_{1}, \ldots, \alpha_{n} \\
\left|\alpha_{1}\right| \cdots, \cdots,\left|\alpha_{i_{j} \mid=1}\right|}}\left(c^{\alpha_{i_{1}} \alpha_{i_{2}} \cdots \alpha_{i j} j \alpha} D^{\alpha} D^{\alpha_{1}} \cdots D^{\alpha_{n}} \psi,\right. \\
& \left.\times D^{\alpha_{1}} \cdots \hat{D}^{\alpha_{i_{1}}} \ldots \hat{D}^{\alpha_{i_{j}}} \ldots D^{\alpha_{n} \eta}\right)_{0}
\end{aligned}
$$

for any $\psi \in W_{2}^{n+1}\left(R^{d}\right)$ and $\eta \in W_{2}^{n}\left(R^{d}\right)$, where the functions $b^{\alpha_{i_{1} \alpha_{2}} \cdots \alpha_{i j} \alpha \beta}$ and $c^{\alpha_{i_{1}} \alpha_{i_{2}} \cdots \alpha_{i} j_{\alpha} \alpha}$ have derivatives in $x$ up to order $k$ inclusive uniformly bounded with respect to $t, x, \omega, \alpha, \beta$ and $\alpha_{i}, a_{t}^{i \alpha}$ are the $i$-th components of the vectors $a_{t}^{\alpha}$, and $(\cdot, \cdot)_{0}$ denotes the inner product of $L^{2}\left(R^{d}\right)$.

Now we are in position to prove Theorem 1.

Proof of Theorem 1. Put $H=W_{2}^{n+k}\left(R^{d}\right)$ and $V=W_{2}^{n+k+1}\left(R^{d}\right)$. In order to appeal to the Krylov-Rozovskii's result, we will introduce operators $\boldsymbol{A}_{t}$ and $\boldsymbol{B}_{t}$. Let us define as follows:

$$
\begin{aligned}
& \left\langle A_{t}^{\prime} \psi, \eta\right\rangle=\left(a_{t}^{\alpha \beta} D^{\beta} D^{\alpha_{1}} \cdots D^{\alpha_{n}} \psi,(-1)^{|\alpha|} D^{\alpha} D^{\alpha_{1}} \cdots D^{\alpha_{n}} \eta\right)_{k}
\end{aligned}
$$

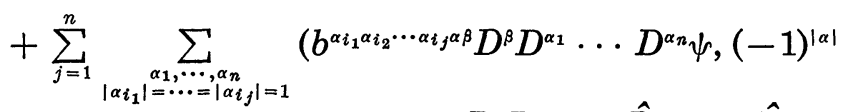

$$
\begin{aligned}
& \left.\times D^{\alpha} D^{\alpha_{1}} \cdots \hat{D}^{\alpha_{i_{1}}} \cdots \hat{D}^{\alpha_{i_{j}}} \cdots D^{\alpha_{n}} \eta\right)_{k}, \\
& \left\langle A_{t} \psi, \eta\right\rangle=\left\langle A_{t}^{\prime} \psi, \eta\right\rangle+\left(\Lambda^{-1} f_{t}, \eta\right)_{n+k+1}
\end{aligned}
$$

and

$$
\begin{aligned}
& \left(\boldsymbol{B}_{t}^{i} \psi, \eta\right)_{H}=\left(a_{t}^{i \alpha} D^{\alpha} D^{\alpha_{1}} \cdots D^{\alpha_{n}} \psi, D^{\alpha_{1}} \cdots D^{\alpha_{n}} \eta\right)_{k} \\
& +\sum_{j=1}^{n} \sum_{\substack{\alpha_{1} \\
\left|\alpha_{i_{1}}\right|, \cdots, \ldots=\left|\alpha_{i_{j} \mid}\right|=1}}\left(c^{\alpha_{i_{1}} \alpha_{\alpha_{2}} \cdots \alpha_{i} j \alpha} D^{\alpha} D^{\alpha_{1}} \cdots D^{\alpha_{n}} \psi\right. \\
& \left.\times D^{\alpha_{1}} \cdots \hat{D}^{\alpha_{i_{1}}} \cdots \hat{D}^{\alpha_{i_{j}}} \cdots D^{\alpha_{n} \eta}\right)_{k}
\end{aligned}
$$

for any $\psi$ and $\eta$ in $V$, where $a_{t}^{i \alpha}, b^{\alpha_{i_{1}} \alpha_{2} \cdots \alpha_{i_{j} \alpha \beta}}$ and $c^{\alpha_{t_{1}} \alpha_{i_{2}} \cdots \alpha_{t_{j}} j_{\alpha}}$ are the functions which appeared in Lemma.

*) The set $\left\{i_{1}, i_{2}, \cdots, i_{j}\right\}$ is contained in the set $\{1,2,3, \cdots, n\}$. 
Taking into consideration that the inequality

$$
\left.\left|\left\langle A_{t} \psi, \eta\right\rangle\right| \leqq \text { const. }\|\psi\|_{V}+\left\|f_{t}\right\|_{n+k-1}\right)\|\eta\|_{V}
$$

holds, and that $n+k-1 \geqq 0$, we see that $A(\psi, t, \omega)=A_{t} \psi$ is a mapping defined on $V$ with values in $V^{*}$ for each $(t, \omega)$. On the other hand, we have

$$
\left|\left(\boldsymbol{B}_{t}^{i} \psi, \eta\right)_{H}\right| \leqq \text { const. }\|\psi\|_{V}\|\eta\|_{H},
$$

hence $\boldsymbol{B}^{i}(\psi, t, \omega)=\boldsymbol{B}_{t}^{i} \psi$ is a mapping from $V$ into $H$ for each $(t, \omega)$.

Now, let us consider the following stochastic equation

$$
\left(\psi_{t}, \eta\right)_{H}=(\gamma, \eta)_{H}+\int_{s}^{t}\left\langle A_{\tau} \psi_{\tau}, \eta\right\rangle d \tau+\int_{s}^{t}\left(B_{\tau} \psi_{\tau}, \eta\right)_{H} d B_{\tau} .
$$

Equation (2.4) is equivalent to the next equation

$$
\begin{aligned}
\left(\psi_{t}, \eta\right)_{n+k}= & (\gamma, \eta)_{n+k}+\int_{s}^{t}\left\langle\boldsymbol{A}_{\tau}^{\prime} \psi_{\tau}, \eta\right\rangle d \tau+\int_{s}^{t}\left(\boldsymbol{B}_{\tau} \psi_{\tau}, \eta\right)_{n+k} d B_{\tau} \\
& +\int_{s}^{t}\left(f_{\tau}, \eta\right)_{n+k} d \tau
\end{aligned}
$$

because

$$
\int_{s}^{t}\left(\Lambda^{-1} f_{\tau}, \eta\right)_{n+k+1} d \tau=\int_{s}^{t}\left(f_{r}, \eta\right)_{n+k} d \tau, \quad t \in[s, T] \quad \text { and } \eta \in V,
$$

with probability 1 under the condition $f . \in \hat{H}_{2}^{n+k}(s, T)$. In order to show that Equation (2.4) has a unique solution in $L^{2}([s, T] \times \Omega, V) \cap L^{2}(\Omega$, $C([s, T] ; H)$ ), we will make use of the above-mentioned Krylov-Rozovskii's result.

In our case, the condition (A, I) is obvious. To check (A, II)-(A, IV), we will calculate the quantities $\left\langle\boldsymbol{A}_{t}^{\prime} \psi, \psi\right\rangle$ and $\sum_{i=1}^{d_{1}}\left\|\boldsymbol{B}_{t}^{i} \psi\right\|_{H}^{2}$.

$$
\begin{aligned}
& \left\langle A_{t}^{\prime} \psi, \psi\right\rangle \leqq\left(a_{t}^{\alpha \beta} D^{\beta} D^{\alpha_{1}} \cdots D^{\alpha_{n}} \psi,(-1)^{|\alpha|} D^{\alpha} D^{\alpha_{1}} \cdots D^{\alpha_{n}} \psi\right)_{k}+c_{1}\|\psi\|_{V}\|\psi\|_{H} \\
& \leqq-\sum_{|\alpha|=|\beta|=1}\left(a_{t}^{\alpha \beta} D^{\gamma_{1}} \cdots D^{\gamma_{k}} D^{\beta} D^{\alpha_{1}} \cdots D^{\alpha_{n}} \psi\right. \text {, } \\
& \left.\times D^{\gamma_{1}} \cdots D^{\gamma_{k}} D^{\alpha} D^{\alpha_{1}} \cdots D^{\alpha_{n}} \psi\right)_{0} \\
& +c_{2}\|\psi\|_{V}\|\psi\|_{H} \\
& \leqq-\sum_{|\alpha|=|\beta|=1}\left(a_{t}^{\alpha \beta} D^{\beta} D^{\gamma_{1}} \cdots D^{\gamma_{k}} D^{\alpha_{1}} \cdots D^{\alpha_{n}} \psi\right. \text {, } \\
& \left.\times D^{\alpha} D^{\gamma_{1}} \cdots D^{\gamma_{k}} D^{\alpha_{1}} \cdots D^{\alpha_{n}} \psi\right)_{0} \\
& +c_{2} \varepsilon\|\psi\|_{V}^{2}+c_{2} \frac{1}{\varepsilon}\|\psi\|_{H}^{2},
\end{aligned}
$$


where $\varepsilon$ is an arbitrary positive number.

On the other hand, we have

$$
\begin{aligned}
& \left|\left(\boldsymbol{B}_{t}^{i} \psi, \eta\right)_{H}\right| \leqq\left|\left(a_{t}^{i \alpha} D^{\alpha} D^{\gamma_{1}} \cdots D^{\gamma_{k}} D^{\alpha_{1}} \cdots D^{\alpha_{n}} \psi, D^{\gamma_{1}} \cdots D^{\gamma_{k}} D^{\alpha_{1}} \cdots D^{\alpha_{n}} \eta\right)_{0}\right| \\
& +\sum_{j=1}^{n} \sum_{\substack{\alpha_{1}, \cdots, \alpha_{n} \\
\left|\alpha_{i_{1}}\right|=\cdots=\left|\alpha_{i j}\right|=1}} \mid\left(c^{\alpha_{i_{1}} \alpha_{i_{2}} \cdots \alpha_{i_{j} i \alpha}} D^{\alpha} D^{\gamma_{1}} \cdots D^{\gamma_{k}} D^{\alpha_{1}} \cdots D^{\alpha_{n}} \psi\right. \text {, } \\
& \left.\times D^{\gamma_{1}} \ldots D^{\gamma_{k}} D^{\alpha_{1}} \ldots \hat{D}^{\alpha_{i_{1}}} \ldots \hat{D}^{\alpha_{i_{j}}} \ldots D^{\alpha_{n}} \eta\right)_{0} \\
& +c_{3}\|\psi\|_{H}\|\eta\|_{H} \text {. }
\end{aligned}
$$

Noting the $n+k+1$ times differentiability of the coefficients $a_{t}^{i \alpha}$, we see that the right hand side of (2.6) is not greater than

$$
\begin{aligned}
& \sum_{|\alpha|=1}\left|\left(a_{t}^{i \alpha} D^{\alpha} D^{\gamma_{1}} \cdots D^{\gamma_{k}} D^{\alpha_{1}} \cdots D^{\alpha_{n}} \psi, D^{r_{1}} \cdots D^{r_{k}} D^{\alpha_{1}} \cdots D^{\alpha_{n}} \eta\right)_{0}\right| \\
& +\sum_{j=1}^{n} \sum_{\substack{\alpha_{1}, \ldots, \alpha_{n} \\
\left|i_{i_{1} \mid}\right|=\ldots \ldots=\left|\alpha_{i_{j} \mid=1}\right|=1}} \mid\left(D^{\gamma_{1}} \cdots D^{\gamma_{k}} D^{\alpha_{1}} \cdots D^{\alpha_{n}} \psi\right. \\
& \left.\times c^{\alpha_{i_{1}} \alpha_{i_{2}} \cdots \alpha_{i j} i \alpha} D^{\alpha} D^{\gamma_{1}} \cdots D^{\gamma_{k}} D^{\alpha_{1}} \cdots \hat{D}^{\alpha_{i_{1}}} \cdots \hat{D}^{\alpha_{i_{j}}} \cdots D^{\alpha_{n}} \eta\right)_{0} \mid \\
& +c_{4}\|\psi\|_{H}\|\eta\|_{H} \\
& \leqq \sum_{|\alpha|=1}\left\|a_{t}^{i \alpha} D^{\alpha} D^{\gamma_{1}} \cdots D^{\gamma_{k}} D^{\alpha_{1}} \cdots D^{\alpha_{n}} \psi\right\|_{0}\|\eta\|_{H}+c_{5}\|\psi\|_{H}\|\eta\|_{H} \cdot
\end{aligned}
$$

Here, $\|\cdot\|_{0}$ denotes the norm of the space $L^{2}\left(R^{d}\right)$. Making use of CauchySchwartz-Bunjakovskii's inequality

$$
(a+b)^{2} \leqq(1+\varepsilon) a^{2}+\left(1+\frac{1}{\varepsilon}\right) b^{2}, \quad \varepsilon>0,
$$

we have

$$
\begin{aligned}
\left\|\boldsymbol{B}_{t}^{i} \psi\right\|_{H}^{2} \leqq & (1+\varepsilon)\left\|a_{t}^{i \alpha} D^{\alpha} D^{\gamma_{1}} \cdots D^{\gamma_{k}} D^{\alpha_{1}} \cdots D^{\alpha_{n}} \psi\right\|_{0}^{2} \\
& +c_{5}\left(1+\frac{1}{\varepsilon}\right)\|\psi\|_{H}^{2} \\
\leqq & \sum_{|\alpha|=1}\left\|a_{t}^{i \alpha} D^{\alpha} D^{\gamma_{1}} \cdots D^{\gamma_{k}} D^{\alpha_{1}} \cdots D^{\alpha_{n}} \psi\right\|_{0}^{2} \\
& +c_{6} \varepsilon\|\psi\|_{V}^{2}+c_{7}(\varepsilon)\|\psi\|_{H}^{2}
\end{aligned}
$$

and hence

$$
\begin{aligned}
\sum_{i=1}^{d_{1}}\left\|B_{t}^{i} \psi\right\|_{H}^{2} \leqq & \sum_{|\alpha|=1}\left\|\left|a_{t}^{\alpha} D^{\alpha} D^{\gamma_{1}} \cdots D^{\gamma_{k}} D^{\alpha_{1}} \cdots D^{\alpha_{n}} \psi\right|\right\|_{0}^{2} \\
& +c_{8} \varepsilon\|\psi\|_{V}^{2}+c_{9}(\varepsilon)\|\psi\|_{H}^{2} .
\end{aligned}
$$

Let $\varepsilon$ in (2.5) and (2.7) be sufficiently small, then we get the next inequality from (2.5), (2.7) and the assumption B) in $\S 1$ : 


$$
2\left\langle\boldsymbol{A}_{t}^{\prime} \psi, \psi\right\rangle+\sum_{i=1}^{d_{1}}\left\|\boldsymbol{B}_{t}^{i} \psi\right\|_{H}^{2} \leqq-\delta^{\prime}\|\psi\|_{V}^{2}+c^{\prime}\|\psi\|_{H}^{2}
$$

holds for any $\psi$ in $V$, where $\delta^{\prime}$ and $c^{\prime}$ are positive constants.

By (2.8), it is easily verified that the inequality

$$
\begin{gathered}
2\left\langle\boldsymbol{A}_{t} \psi_{1}-A_{t} \psi_{2}, \psi_{1}-\psi_{2}\right\rangle+\sum_{i=1}^{d_{1}}\left\|\boldsymbol{B}_{t}^{i} \psi_{1}-\boldsymbol{B}_{t}^{i} \psi_{2}\right\|_{H}^{2} \\
\leqq c^{\prime}\left\|\psi_{1}-\psi_{2}\right\|_{H}^{2}
\end{gathered}
$$

holds for any $\psi_{1}$ and $\psi_{2}$ in $V$. That is, the condition (A, II) is verified.

Noting the inequality

$$
\begin{aligned}
\left|\left(\Lambda^{-1} f_{t}, \psi\right)_{n+k+1}\right| & \leqq\left\|\Lambda^{-1} f_{t}\right\|_{V}\|\psi\|_{V} \\
& \leqq\left\|f_{t}\right\|_{n+k-1}\|\psi\|_{V} \\
& \leqq\left\|f_{t}\right\|_{n+k}\|\psi\|_{V} \\
& \leqq \frac{\varepsilon}{2}\|\psi\|_{V}^{2}+\frac{1}{2 \varepsilon}\left\|f_{t}\right\|_{H}^{2}
\end{aligned}
$$

and choosing a sufficiently small $\varepsilon>0$, we obtain from (2.8)

$$
\begin{aligned}
& 2\left\langle A_{t} \psi, \psi\right\rangle+\sum_{i=1}^{d_{1}}\left\|\boldsymbol{B}_{t}^{i} \psi\right\|_{H}^{2} \\
& \quad \leqq-\delta^{\prime \prime}\|\psi\|_{V}^{2}+c^{\prime \prime}\|\psi\|_{H}^{2}+c^{\prime \prime \prime}\left\|f_{t}\right\|_{H}^{2} .
\end{aligned}
$$

Put $f(t, \omega)=\left\|f_{t}\right\|_{H}^{2}$. Taking into consideration (2.10) and

$$
\left\|A_{t} \psi\right\|^{*} \leqq \text { const. }\|\psi\|_{V}+\left\|f_{t}\right\|_{n+k-1},
$$

we can verify the conditions (A.III) and (A.IV).

Hence the Krylov-Rozovskii's result can be applied to Equation (2.4), and we get that Equation (2.4) has a unique solution. Thus, we see that Equation (2.4) has a solution $\psi$. in the space $\hat{H}_{2}^{n+k+1}(s, T)$.

In order to complete the proof, it is sufficient to show that any solution of Equation (2.4) is a solution of Equation (1.1). Making use of (1.2) and Lemma, we can see that

$$
\left\langle A_{t} \psi, \Lambda^{-k} \eta\right\rangle=\left(D^{\beta} \psi,(-1)^{|\alpha|} a_{t}^{\alpha \beta} D^{\beta} \eta\right)_{n}+\left(f_{t}, \eta\right)_{n}, \quad \psi, \eta \in V,
$$

holds with almost all $(t, \omega)$, and that

$$
\left\langle\boldsymbol{B}_{t} \psi, \Lambda^{-k} \eta\right\rangle=\left(D^{\alpha} \psi, a_{t}^{\alpha} \eta\right)_{n}, \quad \psi, \eta \in V .
$$

Hence, replacing $\eta$ by $\Lambda^{-k} \eta$ in Equation (2.4), we obtain that any solution of Equation (2.4) satisfies Equation (1.1). Thus, the proof is complete. 


\section{§. 3. Proof of Theorem 2}

Proof of Theorem 2. Let $\psi$. be the solution of Equation (1.1) with $f_{t}=0$. That is, $\psi$. satisfies the equation

$$
\left(\psi_{t}, \eta\right)_{n}=(\gamma, \eta)_{n}+\int_{s}^{t}\left(D^{\beta} \psi_{\tau},(-1)^{|\alpha|} a_{\tau}^{\alpha \beta} D^{\alpha} \eta\right)_{n} d \tau+\int_{s}^{t}\left(D^{\alpha} \psi_{\tau}, a_{\tau}^{\alpha} \eta\right)_{n} d B_{\tau} .
$$

Note that $\wedge \psi$. with initial data $\wedge \gamma$ satisfies Equation (0.4). Let $j(t)$ be smooth function such that $j(s)=0$ and $j(t)>0$ for $t>s$. Putting $u_{t}=$ $j(t) \psi_{t}$, we can see by Itô's formula

$$
\begin{aligned}
\left(u_{t}, \eta\right)_{n}= & \int_{s}^{t}\left(D^{\beta} u_{\tau},(-1)^{|\alpha|} a_{\tau}^{\alpha \beta} D^{\alpha} \eta\right)_{n} d \tau+\int_{s}^{t}\left(D^{\alpha} u_{\tau}, a_{\tau}^{\alpha} \eta\right)_{n} d B_{\tau} \\
& +\int_{s}^{t}\left(j^{\prime}(\tau) \psi_{\tau}, \eta\right)_{n} d \tau .
\end{aligned}
$$

Here, we know that $j^{\prime} \psi_{.} \in \hat{H}_{2}^{n+1}(s, T)$ and $u_{s}=0$. Hence, by Theorem 1 , we see that $u=j \psi . \in \hat{H}_{2}^{n+2}(s, T)$. Repeating this argument, we get

$$
\int_{s}^{t} j(\tau) d \tau \cdot \psi_{.} \in \hat{H}_{2}^{n+3}(s, T)
$$

Finally, we obtain

$$
\int_{s}^{t} \int_{s}^{\tau_{h}} \cdots \int_{s}^{\tau_{2}} j\left(\tau_{1}\right) d \tau_{1} \cdots d \tau_{h} \cdot \psi_{.} \in \hat{H}_{2}^{m+1}(s, T), \quad h=m-n-1 .
$$

Thus, we see that $\psi_{t} \in W_{2}^{m}\left(R^{d}\right)$ for each $(t, \omega) \in[s, T] \times \Omega$.

Finally, we will prove Corollary to Theorem 2.

Proof of Corollary to Theorem 2. Under the assumptions of Corollary, we see by Theorem 2 and Proposition 2 that Equation (0.4) has a unique solution $\psi_{.} \in \hat{H}_{2}^{l}(s, T)$ satisfying $\psi_{t}(x, \omega) \in W_{2}^{m-2}\left(R^{d}\right)$ for each $(t, \omega) \in(s, T] \times$ $\Omega$. Put $\phi(s, z, t, x ; \omega)=(I-\Delta)^{l} \psi_{t}=\Lambda^{l} \psi_{t}$. Then, $\phi(s, z, t, x ; \omega) \in W_{2}^{m-2 n}\left(R^{d}\right)$ for each $(s, z, t, \omega)$ as a function of $x$. Noting (1.2), we have

$$
\Phi_{t}[\eta]=\left(\psi_{t}, \eta\right)_{\ell}=\left(\Lambda^{-\ell} \phi, \eta\right)_{\ell}=(\phi, \eta)_{0}=\int_{r^{d}} \phi(s, z, t, x ; \omega) \eta(x) d x
$$

Thus, the proof is complete.

\section{REFERENCES}

[1] M. Fujisaki, G. Kallianpur and H. Kunita, Stochastic differential equations for the non-linear filtering problem, Osaka J. Math., 9 (1972), 19-42.

[2] N. V. Krylov and B. L. Rozovskii, On Cauchy problem for linear stochastic partial 
differential equations, Izv. Akad. Nauk SSSR, Ser. Mat., 41 (1977), 1329-1347; English transl. in Math. USSR Izv., 11 (1977).

[ 3 ] N. V. Krylov and B. L. Rozovskii, On conditional distributions of diffusion processes, Izv. Akad. Nauk SSSR, Ser. Mat., 42 (1978), 356-378; English transl. in Math. USSR Izv., 12 (1978).

[4] — Itô's equations in Banach spaces and strongly parabolic stochastic partial differential equations, Doklady Akad. Nauk, 249 (1979), 285-289.

[5] — On stochastic evolution equations, Sovremennye problemy matematiki, 14, Itogi nauki i tekhniki (1979).

[ 6 ] R. S. Liptser and A. N. Shiryayev, Statistics of random processes, Nauka, Moscow, 1974; English transl. Vols. I, II, Springer-Verlag, 1977.

[ 7 ] H. P. Mckean, Jr., Stochastic integrals, Academic press, 1969.

[ 8 ] L. Nirenberg, On elliptic partial differential equations, Ann. Scuola Norm. Sup. Pisa, 13 (1959), 115-162.

[ 9 ] E. Pardoux, Filtrage de diffusions avec conditions frontières, caractérisation de la densité conditionelle, Journées de Statistique des Processus Stochastiques, Lecture Notes in Mathematics, 636, Springer-Verlag (1977), 163-188.

В. L. Rozovskii (Б. Л. Розовский)

Московский Институт Повышения Квалификации

Руководящих Работников и Специалистов

Химицеской Промышленности, Москва, СССР

A. Shimizu

Nagoya Institute of Technology

Nagoya 466, Japan 